\title{
Pyomyositis in a Patient Undergoing Chemotherapy for Gastric Cancer: A Case Report and Literature Review
}

\author{
Yoko Nakayama $^{a}$ Akira Sugiyama $^{a}$ Tetsu Yamamoto $^{b}$ \\ Ryoji Hyakudomi $^{\text {b }}$ Noriyuki Hirahara ${ }^{b}$ Yoshitsugu Tajima ${ }^{b}$ \\ ${ }^{a}$ Department of Surgery, Izumo City General Medical Center, Izumo, Japan; bepartment of \\ Digestive and General Surgery, Shimane University Faculty of Medicine, Izumo, Japan
}

\section{Keywords}

Pyomyositis · Gastric cancer · Chemotherapy · Irinotecan · Antibiotics

\begin{abstract}
Pyomyositis has recently been recognized as a primary infection of the large skeletal muscles, and it is often accompanied by single or multiple intramuscular abscess formation. Immunocompromised patients, including those with diabetes mellitus, human immunodeficiency virus infection, and cancer, as well as those undergoing chemotherapy, are at a greater risk of developing pyomyositis. A 78-year-old Japanese man with recurrent gastric cancer being treated with chemotherapy presented with sudden-onset pain in his left lower extremity while undergoing a second-line regimen with irinotecan. T2-weighted magnetic resonance imaging (MRI) showed an abnormally high-intensity signal in the left internal and external obturator muscles, a finding consistent with pyomyositis. Following intensive antibiotic treatment, the patient recovered completely and was able to resume chemotherapy with irinotecan. For a patient who developed pyomyositis during chemotherapy for gastric cancer, early diagnosis using MRI followed by administration of timely intensive antibiotic therapy resulted in complete recovery.
\end{abstract}

\section{Introduction}

Pyomyositis is a purulent infection involving the large skeletal muscles, is usually caused by Staphylococcus aureus, and often presents with single or multiple intramuscular abscesses $[1,2]$. Although pyomyositis is classically a tropical disease, it is being increasingly recognized in temperate climates and encountered particularly in immunocompromised patients, such 
as those with diabetes mellitus, human immunodeficiency virus infection, autoimmune disorders, chronic liver diseases, rheumatologic conditions, intravenous drug addiction, and malignancies, especially in those undergoing chemotherapy [1,3]. A definitive diagnosis of pyomyositis can be difficult because of its rare occurrence and varied clinical manifestations; however, a delay in diagnosis can result in serious complications, including multiple abscess formation, septicemia, multi-organ system failure, and occasionally death $[1,4,5]$.

Although pyomyositis has recently been recognized as a rare but important complication in patients with hematological malignancies undergoing chemotherapy [3], to the best of our knowledge, only 10 such cases in patients with solid tumors undergoing chemotherapy have been reported [6-14]. A patient with advanced pyomyositis, requiring interventional treatment with percutaneous or operative drainage and debridement, may have to defer or interrupt a potentially life-saving chemotherapeutic regimen. Chemotherapy was resumed only in 2 of the 10 previously reported cases. Therefore, early diagnosis and effective treatment of pyomyositis in patients with malignant tumors during chemotherapy are crucial. Here, we report the first case of pyomyositis detected at an early stage during chemotherapy for gastric cancer; this is the only reported case of solid cancer in which chemotherapy was continued without recurrence of pyomyositis after 22 months of onset.

\section{Case Report/Case Presentation}

A 78-year-old Japanese man, who underwent 13 cycles of second-line chemotherapy with irinotecan for recurrent gastric cancer, was urgently admitted to our hospital with a chief complaint of sudden-onset, movement-related pain in his left lower extremity associated with a high-grade fever of $38.3^{\circ} \mathrm{C}$. After completing his last 12-day cycle of chemotherapy, he experienced a transient episode of nausea and high-grade fever, and severe manifestations subsequently occurred 2 days later.

The patient had undergone a distal gastrectomy, with D2 lymphadenectomy and a Rouxen-Y reconstruction, for gastric cancer in December 2015. Histopathological examination of the resected specimens revealed a human epidermal growth factor receptor-2-positive, papillary adenocarcinoma of the stomach, further classified as T2N1M0, Stage IIA based on the Union for International Cancer Control TNM classification. Adjuvant chemotherapy with tegafur, gimeracil, and oteracil (TS-1) was planned to be administered to the patient for 1 year after the diagnosis. However, at 7 months after adjuvant chemotherapy with TS-1, left supraclavicular and multiple para-aortic lymph-node metastases were detected on the follow-up computed tomography (CT) scan. Thus, he was administered combination chemotherapy with capecitabine, cisplatin, and trastuzumab for 6 months. However, his left supraclavicular and multiple para-aortic lymph-node metastases worsened; therefore, he was administered chemotherapy with irinotecan, after which he showed stable disease. The patient was taking oral medications for diabetes mellitus and hypertension while receiving chemotherapy; thus, the disorders were well managed and controlled.

Physical examination on admission did not reveal any signs of erythema, swelling, or tenderness in the left lower extremity. However, blood tests demonstrated leukocytosis, with a white blood cell count of $12,500 / \mu \mathrm{L}$ (normal range, 4,000-9,000/ $\mu \mathrm{L}$ ) and a left shift of 95.5\%. In addition, the C-reactive protein level had significantly increased to $20.6 \mathrm{mg} / \mathrm{dL}$ (normal range, $0-0.3 \mathrm{mg} / \mathrm{dL}$ ). Liver enzymes were slightly elevated, with aspartate transaminase, alanine transaminase, and gamma-glutamyl transpeptidase levels at $57 \mathrm{IU} / \mathrm{L}$ (normal range, 8-40 IU/L), $87 \mathrm{IU} / \mathrm{L}$ (normal range, 5-39 IU/L), and $326 \mathrm{IU} / \mathrm{L}$ (normal range, 10-47 IU/L), respectively. Serum lactic acid dehydrogenase and creatine kinase levels were within normal limits.

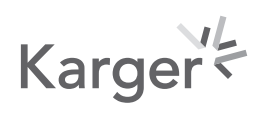


An enhanced CT scan of the abdomen and pelvis did not show any remarkable changes, such as intramuscular abscess or hematoma development, thrombophlebitis, fracture, or metastatic tumors (shown in Fig. 1a, b). There was no evidence of bacteremia or urinary tract infection in either blood or urine cultures. A biliary tract infection complicated with pseudogout of the left hip joint was suspected, and the patient was treated empirically with intravenous broad-spectrum antibiotics consisting of a piperacillin-tazobactam combination (4.5 $\mathrm{g} / 8 \mathrm{~h}$ ) and oral acetaminophen $(1,200 \mathrm{mg} /$ day $)$.

After initiating antibiotic treatment, fever and inflammatory reactions decreased. However, the patient continued to complain of persistent pain in the left thigh. Magnetic resonance imaging (MRI) examination of the pelvis, performed on the sixth day of hospitalization, revealed marked enlargement of the left internal and external obturator, pectineus, and adductor muscles on T1-weighted images (shown in Fig. 2a). The affected muscles showed an abnormally high signal intensity on T2-weighted (shown in Fig. 2b) and short tau inversion recovery sequence images (shown in Fig. 2c). These findings were consistent with a diagnosis of pyomyositis. The patient's left lower extremity pain improved after 12 days of intravenous antibiotic treatment with piperacillin-tazobactam. The patient was discharged and received oral cefdinir $(100 \mathrm{mg} / 6 \mathrm{~h}$ ) for an additional 4 weeks.

At the 7-week follow-up, an MRI scan showed complete resolution of the previous signs of pyomyositis (shown in Fig. 2d-f). The patient's full range of left hip motion was restored without any lasting sequelae. Therefore, he resumed chemotherapy with irinotecan, which was administered for an additional 8 cycles. At the 22-month follow-up after discharge, while undergoing fifth-line chemotherapy for newly developed lesions in the liver and lung, the patient showed no signs of recurrent pyomyositis.

\section{Discussion/Conclusion}

Pyomyositis is a primary acute infection of skeletal muscles and is often associated with abscess formation [1,2]. In 12-40\% of cases, patients had multiple abscesses, and the most common lesions were in the quadriceps muscles, followed by bulky muscles such as the gluteal muscles [4]. Skeletal muscle tissue is naturally resistant to infection. Therefore, it has been suggested that pyomyositis is induced by local mechanical trauma, parasitic infection, malnutrition, and a compromised immune system [5]. Our patient had diabetes mellitus and relapsing gastric cancer, and he was undergoing chemotherapy for the latter diagnosis, which may have increased his susceptibility to pyomyositis. Walling et al. reported that $15 \%$ of pyomyositis patients had diabetes mellitus [15]. It has been reported that skeletal muscle damage is caused by muscle infarction, even without frank infarction, local vascular insufficiency, and hypoxia due to diabetic microvascular diseases [15]. Development of pyomyositis in patients with neoplastic disease after chemotherapy is usually ascribed to neutropenia and/or immunodeficiency, caused by cancer. However, subclinical myopathy secondary to malignancy and/or its treatment is another possible cause [4]. Several chemotherapeutic drugs have been reported to induce muscle toxicity-related pyomyositis [9], although irinotecan muscle toxicity has never been reported. Notwithstanding, the exact pathogenesis is currently poorly understood; our patient had several pyomyositis risks, such as diabetes mellitus and cancer, and was undergoing chemotherapy.

Pyomyositis has 3 clinical stages $[1,4,5]$, including the invasive stage, which is characterized by an onset of manifestations, including low-grade fever, localized swelling, mild pain, and tenderness of the affected muscle. Typically, pus cannot be aspirated from the affected site at this stage. Next is the suppurative stage, when most patients present with high-grade fever, distinct muscle swelling, and tenderness. Aspiration of pus from the infected site can

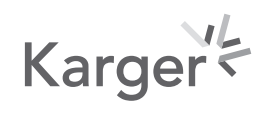




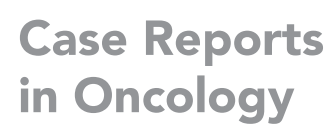

Case Reports in Oncology
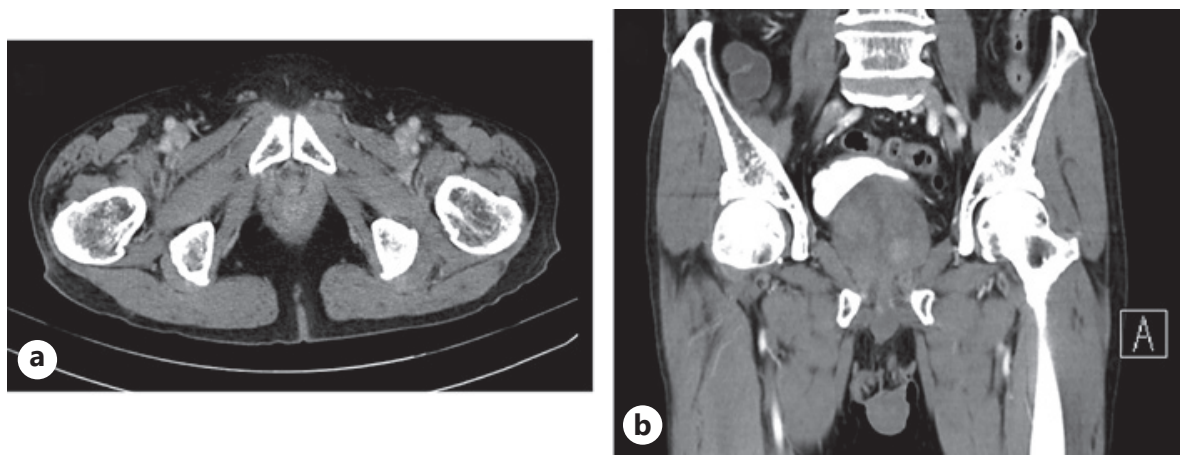

Fig. 1. Enhanced CT scan of the pelvis. Axial (a) and coronal (b) enhanced CT imaging showing no remarkable changes such as intramuscular abscesses, hematoma development, or intravascular defects. CT, computed tomography.
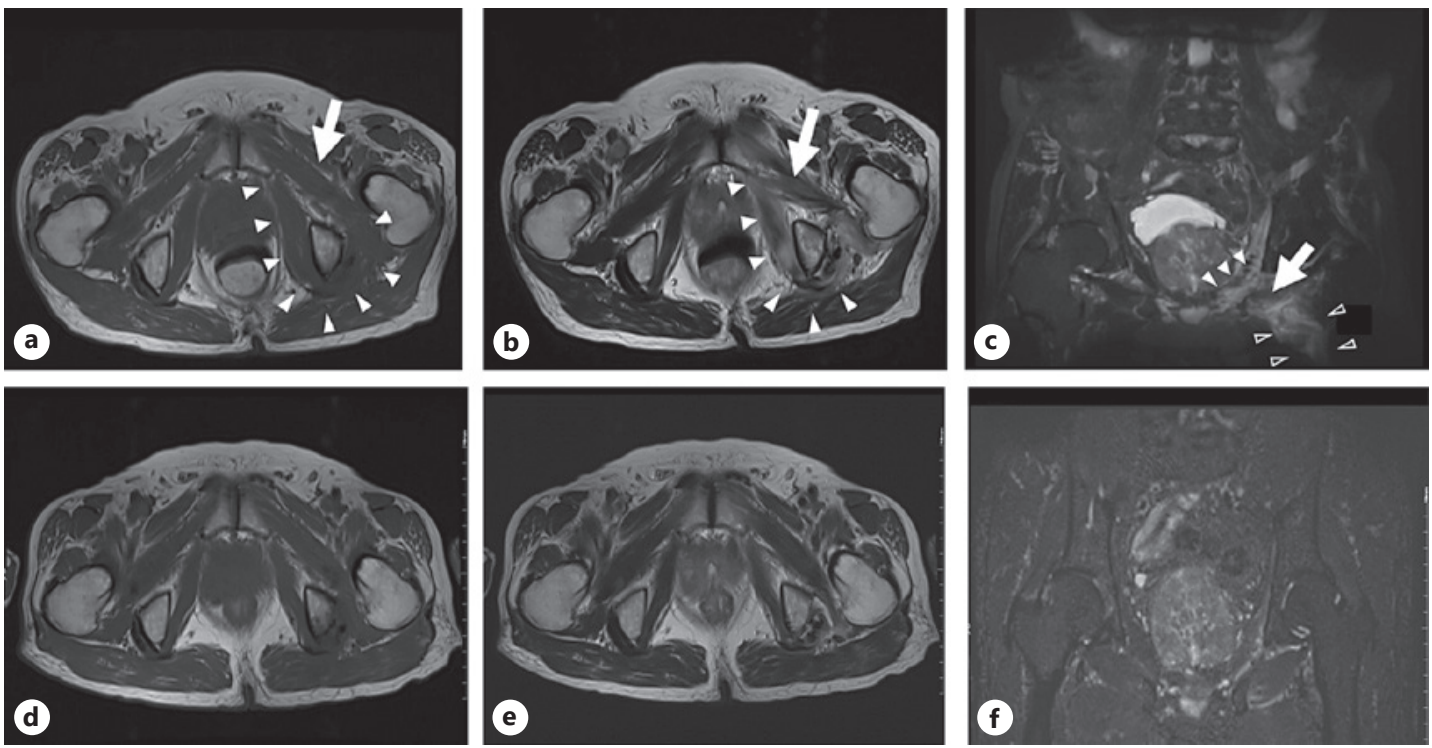

Fig. 2. MRI observations of the pelvis. The first line shows the onset (a-c), while the second line shows the seventh week after the treatment (d-f). a Axial T1-weighted image showing diffuse enlargement of the left internal (white arrowheads) and external obturator (arrow) muscles with intermediate signal intensity. b Axial T2-weighted image showing a high signal intensity in the left internal (white arrowheads) and external obturator muscles (arrow) with no evidence of an abscess, soft tissue gas, or tumor development. c A coronal short tau inversion recovery sequence image showing a high signal intensity in the left internal (white arrowheads) and external obturator (arrow) and adductor muscles (open arrowheads). d Axial T1-weighted image showing improvement of the diffuse enlargement. e Axial T2-weighted image showing a change in the signal intensity. $\mathbf{f}$ A coronal short tau inversion recovery sequence image showing the disappearance of the high signal intensity. MRI, magnetic resonance imaging.

be performed at this stage. In the third stage, that is, the late stage, the systemic manifestations of sepsis, metastatic abscesses, and multi-organ dysfunction occur [4, 6].

Because of lack of awareness, nonspecific features, and atypical presentations, a wide range of differential diagnoses are often considered such as septic arthritis, cellulitis, muscle hematoma, deep venous thrombosis, osteosarcoma, and polymyositis. Thus, the correct diagnosis is often missed, especially in the early stage $[1,5]$. Various noninvasive diagnostic modalities, such as ultrasonography, CT, and MRI, are useful in confirming the diagnosis of 


\section{Case Reports in Oncology}

Case Rep Oncol 2021;14:1220-1227 DOI: $10.1159 / 000518242$

(c) 2021 The Author(s). Published by S. Karger AG, Basel www.karger.com/cro

Nakayama et al.: Pyomyositis during Gastric Cancer chemotherapy

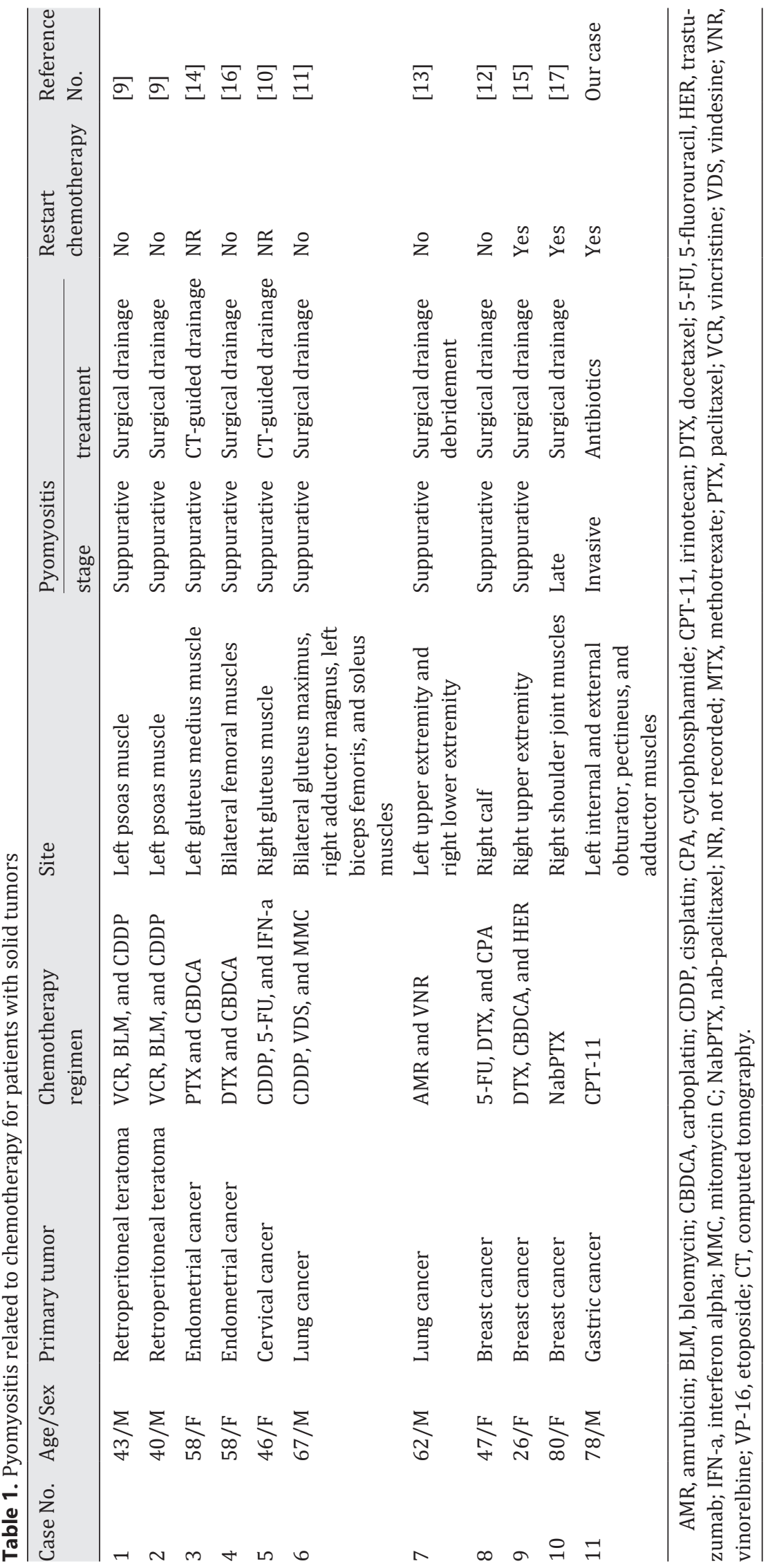


pyomyositis $[1,2,4,5]$. Some studies suggest that MRI is the most useful modality to diagnose pyomyositis at an early stage because it can demonstrate both the site and extent of inflammatory muscle involvement [5].

In these patients, the affected muscles are usually enlarged, showing a homogeneous intermediate signal intensity on T1-weighted MRI studies [2]. T2-weighted MRI may reveal diffuse muscle enlargement with hyperintensity, which may be associated with swelling of fascial planes, an abnormal signal intensity shown by the adjacent bone, or with fluid accumulation in the involved joint $[1,2,16]$. A CT scan can be performed more easily than an MRI, but the former imaging technique has lower sensitivity and provides lesser anatomical information than the latter [16]. For our patient, an enhanced CT scan was performed when he was admitted with leg pain and high fever, but it showed no remarkable changes. MRI examination was performed on the sixth day after admission, which led to a diagnosis of pyomyositis.

Pyomyositis is a rare complication in patients undergoing chemotherapy for cancer. To the best of our knowledge, only 10 patients with solid tumors, who were undergoing chemotherapeutic regimens, have been diagnosed with pyomyositis (Table 1). While the majority of previously reported cases of pyomyositis were diagnosed at the suppurative or late stage with single or multiple muscle abscesses requiring surgical or CT-guided drainage, our patient was diagnosed with pyomyositis at an early stage, using an MRI study. As abscess formation had not yet occurred, the patient could be treated successfully with antibiotics without surgical intervention.

The National Comprehensive Cancer Network clinical practice guidelines for prevention and treatment of cancer-related infections recommend that a patient diagnosed with a condition causing complex bacteremia, such as pyomyositis, should receive antibiotic therapy for 7-14 days, although the regimen may need modification on a case-to-case basis $[17,18]$. As $S$. aureus is the most common causative organism [1], the timely administration of a broadspectrum antibiotic agent providing $S$. aureus coverage is usually sufficient to manage the infection at an early stage empirically, because blood cultures exhibit positivity in only $30 \%$ of cases [2]. Once the abscess has formed, however, interventional treatment with percutaneous or surgical drainage and debridement should be performed on an emergency basis to ensure complete recovery $[1,5]$. In the present case, blood culture was negative; however, administration of piperacillin-tazobactam was effective, and inflammation was controlled. There was no recurrence of pyomyositis after switching to oral cefdinir.

The present case was diagnosed and treated at an early stage, without surgical treatment. Purulent myositis did not recur, although the tumor development gradually progressed, and chemotherapy is being continued to date. McRae and Sharma [12] and Kitayama et al. [14] reported that it was possible to resume chemotherapy after treatment for myositis in patients who required surgical treatment; however, McRae and Sharma [12] reported resuming chemotherapy only once, and Kitayama et al. [14] did not clearly state the time periods. In contrast, Keith and Bramwell [9] and Yamada et al. [10] reported that they were unable to resume chemotherapy, and their patients died due to primary cancer progression. Chemotherapy could be resumed in the case of upper extremity muscle inflammation, but those in the lower extremity were not resumed. Muscle trauma might cause pyomyositis $[1,4,5]$. Surgical treatment for pyomyositis in the bulky muscle, especially in the lower extremity rather than the upper extremity, may form a new onset site and prolong the inflammatory response. This may be one of the reasons why chemotherapy cannot usually be resumed. In addition, infection during chemotherapy may affect prognosis [19], and further, an inflammatory response may affect the pharmacodynamics of anticancer drugs [20]. Therefore, early diagnosis and treatment without surgical intervention for pyomyositis leads to the resumption of treatment for cancer by controlling infection and inflammatory response; this may improve the prognosis of patients.

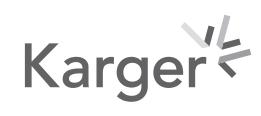


In summary, pyomyositis is a rare complication of chemotherapy in patients with a solid malignancy. When a patient undergoing chemotherapy complains of localized muscular pain, an MRI study should be performed promptly, as early detection and treatment are key to achieving a better prognosis in case of pyomyositis. We suggest that timely diagnosis and effective treatment of pyomyositis can enable the patient to resume chemotherapy early and, thereby, control the progression of a life-threatening malignancy.

\section{Statement of Ethics}

Written informed consent was obtained from the patient for the publication of this case report and any accompanying images. A copy of the written consent is available for review by the editor-in-chief of this journal. The local Ethical Committee of the Izumo City General Medical Center in Shimane, Japan, provided approval for the publication of this case report (Receipt No: 3-1).

\section{Conflict of Interest Statement}

The authors have no conflicts of interest to declare.

\section{Funding Sources}

No funding was received for this report.

\section{Author Contributions}

Y.N. wrote the initial draft. Y.N., T.Y., and A.S. performed the surgery. A.S., T.Y., R.H., N.H., and Y.T. provided valuable guidance and edited the discussion section. All authors read and approved the final draft of the manuscript.

\section{Data Availability Statement}

All data generated or analyzed during this study are included in this article. Further enquiries can be directed to the corresponding author.

\section{References}

1 Agarwal V, Chauhan S, Gupta RK. Pyomyositis. Neuroimaging Clin N Am. 2011 Nov;21(4):975-83.

2 Mitsionis GI, Manoudis GN, Lykissas MG, Sionti I, Motsis E, Georgoulis AD, et al. Pyomyositis in children: early diagnosis and treatment. J Pediatr Surg. 2009 Nov;44(11):2173-8.

3 Chiu SK, Lin JC, Wang NC, Peng MY, Chang FY. Impact of underlying diseases on the clinical characteristics and outcome of primary pyomyositis. J Microbiol Immunol Infect. 2008 Aug;41(4):286-93.

4 Chiedozi LC. Pyomyositis. Review of 205 cases in 112 patients. Am J Surg. 1979 Feb;137(2):255-9.

5 Chauhan S, Jain S, Varma S, Chauhan SS. Tropical pyomyositis (myositis tropicans): current perspective. Postgrad Med J. 2004 May;80(943):267-70.

6 Maguire J, Kaye SB. Psoas abscess: unusual complication of effective chemotherapy for teratoma. Br Med J (Clin Res Ed). 1985 Jun;290(6485):1870.

\section{Karger's}




\section{Case Reports in Oncology}

\begin{tabular}{l|l}
\hline Case Rep Oncol 2021;14:1220-1227 \\
\hline DOI: 10.1159/000518242 & $\begin{array}{l}\text { ○ 2021 The Author(s). Published by S. Karger AG, Basel } \\
\text { www.karger.com/cro }\end{array}$ \\
\hline
\end{tabular}

Nakayama et al.: Pyomyositis during Gastric Cancer chemotherapy

7 Segreti EM, Munkarah A, Levenback C. Successful percutaneous drainage of gluteal pyomyositis occurring after intra-arterial chemotherapy and pelvic irradiation. Gynecol Oncol. 1994 Jun;53(3):376-9.

8 Teramoto S, Konishi M, Mikasa K, Hamada K, Maeda K, Sakamoto M, et al. A case of multiple muscular abscesses of the lower limbs by Staphylococcus aureus after chemotherapy for lung cancer. Kansenshogaku Zasshi. 1997 Sep; 71(9):939-43.

9 Keith BD, Bramwell VH. Pyomyositis after chemotherapy for breast cancer. Am J Clin Oncol. 2000 Feb;23(1): 42-4.

10 Yamada K, Wasa J, Sugiura H, Horio Y. A case of multiple pyomyositis after chemotherapy for lung cancer. Gan to Kagaku Ryoho. 2006 Jun;33(6):837-40.

11 Singh P, Chan W, Blomfield P, McIntosh R. Pyomyositis after chemotherapy for endometrial cancer. Int J Gynecol Cancer. 2010 Oct;20(7):1256-8.

12 McRae M, Sharma S. Forearm pyomyositis in a breast cancer patient on chemotherapy. J Plast Reconstr Aesthet Surg. 2010 Oct;63(10):e737-9.

13 Nakao Y, Yokoyama M, Nishiyama S, Hashiguchi M, Aihara S, Yasunaga M, et al. Pyomyositis associated with chemotherapy for endometrial cancer: a case report. World J Surg Oncol. 2013 Feb;11:45.

14 Kitayama H, Sugiyama J, Hirayama M, Onada Y, Tsuji Y. Shoulder pain after fall, septic shock, and pyomyositis associated with breast cancer chemotherapy and lymphedema. Case Rep Oncol. 2016 Nov;9(3):726-32.

15 Walling DM, Kaelin WG. Pyomyositis in patients with diabetes mellitus. Rev Infect Dis. 1991 Sep;13(5):797802.

16 Kwak YG, Choi SH, Kim T, Park SY, Seo SH, Kim MB, et al. Clinical guidelines for the antibiotic treatment for community-acquired skin and soft tissue infection. Infect Chemother. 2017 Dec;49(4):301-25.

17 National Comprehensive Cancer Network. NCCN guidelines: prevention and treatment of cancer-related infections. Pennsylvania: National Comprehensive Cancer Network; 2018 [cited 2018 0ct 11]. Available from: https://www.nccn.org.

18 Elting LS, Rubenstein EB, Rolston KV, Bodey GP. Outcomes of bacteremia in patients with cancer and neutropenia: observations from two decades of epidemiological and clinical trials. Clin Infect Dis. 1997 Aug;25(2): 247-59.

19 Dendle C, Gilbertson M, Spelman T, Stuart RL, Korman TM, Thursky K, et al. Infection is an independent predictor of death in diffuse large b cell lymphoma. Sci Rep. 2017 Jun;7(1):4395.

20 Slaviero KA, Clarke SJ, Rivory LP. Inflammatory response: an unrecognised source of variability in the pharmacokinetics and pharmacodynamics of cancer chemotherapy. Lancet Oncol. 2003 Apr;4(4):224-32. 